\title{
Large Hadron Collider: The Quest of 'God Particles'
}

\author{
Bhesh Raj Pokharel \\ M.Sc.Physics, P.N.Campus,Pokhara,Email: braj_pokharel@yahoo.com
}

As we know experiment is the sole judge of the scientific truths. In the two hundred years of most rapidly developing field of knowledge in Physics there are many unsolved question and intersecting principles. Many theories about the universe have many unsolved questions like Higgs bosons known as god particles and mystery of the antiparticles etc Thousands of scientists and engineers are now trying to attempt these from the Large Hadron Collider (LHC). Scientists have stepped a next step in the voyage of discovery.

The biggest and most expensive research in the human history, in field of particle physics have started in research named LHC. LHC is the world's large and highest energy particle accelerator. LHC the realm of physics is a gigantic scientific instrument .It has become most expensive scientific experiment with budged of 9 billion US dollar.

LHC lies in a tunnel $27 \mathrm{~km}$ in circumference and 175 meter beneath in the boarder of Switzerland and France near Geneva. It was built by European organization for nuclear research (CERN) . The LHC mainly based on proton-proton collision .The collider tunnel contains two adjacent parallel beams pipes that intersects at four points and is contains the proton beams, which travel in the opposite direction around the beam. Thousands of dipole magnets, quadrapoles magnets and super conducting magnets are used too run the proton beams in circular path and maximize the chance of collisions. The protons take $90 \mu \mathrm{s}$. Around 96 tons of liquid helium is needed to keep the magnets at their operating temperature $1.9 \mathrm{~K}$. The $\mathrm{LHC}$ as well as LHC related simulation will produce a total data output of 15 petabytes per year. Every word spoken worldwide in 1 year converted into text would be 2 to 3 petabytes.

LHC was officially inaugurated in 21 October 2008. For the first time on10 September 2008 the proton beams were successfully circulated in the main ring of the LHC. Due to the serious fault between two super conducting bending magnets operation was halted on 19 September 2008 and proton beam successful circulated again on 20 November 2009. First collision of proton had occurred on 23 November 2009. LHC have resumed the operation on Feb 2010.it will be shut down to repair on 2012 and will start up again at 2013. Due to the faulty electrical connection between two magnets the incident have occurred in which 6 tons of liquid helium which was vested into the tunnel and temperature had risen about $100 \mathrm{~K}$ in some of the affected magnets and a total of 53 magnets were destroyed.

Some are saying that LHC particle collision might produce the microscopic black holes or it may cause the creation of the hypothetical particles called strangelets. But it has examined and confirmed that it has no such danger. LHC was built to help the scientist to answer key unresolved question in particle physics. As we believe from the Big bang theory equal amount of matter and anti matter must have been produced. If so scientist will investigate why this is and what happen to all the anti matter.

Finding the Higgs is one of the main science objectives for the LHC. Higgs boson is the hypothetical massive particles. At present there is no non fundamental scalar particle in nature. The attempts can be made from the LHC. If it is exist it is the integral component of the material world. According to Higgs theory, the particles acquire their masses through their interaction with an all preventing field called Higgs field which is carried by Higgs bosons. LHC will also help to resolve the problems like what is the origin of mass? Why do some particles have no mass at all? Why gravity is weak force than other three fundamental forces? Are various grand unification theories are correct? LHC is the most exciting science adventure of this decade and an important landmark to understand the origin and evolution of the universe, where we live. The successful completion of the LHC will reshape how we think about physical world. It has marked the start of revolutions in particle physics. Scientist will be looking for the new Physics beyond the 'standard model'. טם 